\title{
Small Combinatorial Cardinal Characteristics and Theorems of Egorov and Blumberg
}

Krzysztof Ciesielski

West Virginia University, krzysztof.ciesielski@mail.wvu.edu

Follow this and additional works at: https://researchrepository.wvu.edu/faculty_publications

Part of the Mathematics Commons

\section{Digital Commons Citation}

Ciesielski, Krzysztof, "Small Combinatorial Cardinal Characteristics and Theorems of Egorov and Blumberg" (2000). Faculty Scholarship. 835.

https://researchrepository.wvu.edu/faculty_publications/835 
Real Analysis Exchange

Vol. 26(2), 2000/2001, pp. 905-911

Krzysztof Ciesielski*, Department of Mathematics, West Virginia University,

Morgantown, WV 26506-6310, USA, email: K_Cies@math. wvu.edu, internet:

http: //www . math. wvu. edu/ kcies

Janusz Pawlikowski $\dagger^{\dagger}$ Department of Mathematics, University of Wrocław, pl. Grunwaldzki 2/4, 50-384 Wrocław, Poland, email:

pawlikow@math.uni.wroc.pl and

Department of Mathematics, West Virginia University, Morgantown, WV

26506-6310, USA, email: pawlikow@math.wvu.edu

\title{
SMALL COMBINATORIAL CARDINAL CHARACTERISTICS AND THEOREMS OF EGOROV AND BLUMBERG
}

\begin{abstract}
We will show that the following set theoretical assumption

$\mathfrak{c}=\omega_{2}$, the dominating number $\mathfrak{d}$ equals to $\omega_{1}$, and there

exists an $\omega_{1}$-generated Ramsey ultrafilter on $\omega$

(which is consistent with ZFC) implies that for an arbitrary sequence $f_{n}: \mathbb{R} \rightarrow \mathbb{R}$ of uniformly bounded functions there is a set $P \subset \mathbb{R}$ of cardinality continuum and an infinite $W \subset \omega$ such that $\left\{f_{n} \uparrow P: n \in W\right\}$ is a monotone uniformly convergent sequence of uniformly continuous functions. Moreover, if functions $f_{n}$ are measurable or have the Baire property then $P$ can be chosen as a perfect set.

We will also show that $\operatorname{cof}(\mathcal{N})=\omega_{1}$ implies existence of a magic set and of a function $f: \mathbb{R} \rightarrow \mathbb{R}$ such that $f \uparrow D$ is discontinuous for every $D \notin \mathcal{N} \cap \mathcal{M}$
\end{abstract}

Our set theoretic terminology is standard and follows that of [8]. In particular, $|X|$ stands for the cardinality of a set $X$ and $\mathfrak{c}=|\mathbb{R}|$. We are using

Key Words: cofinality, null sets, uniform convergence, Ramsey ultrafilter, Blumberg theorem, magic set.

Mathematical Reviews subject classification: Primary 26A15, 03E35; Secondary 26A03, $03 \mathrm{E} 17$.

Received by the editors November 17, 2000

* Papers authored or co-authored by a Contributing Editor are managed by a Managing Editor or one of the other Contributing Editors.

†The work of the second author was partially supported by KBN Grant 2 P03A 03114. 
symbols $\mathcal{N}$ and $\mathcal{M}$ to denote the ideals of Lebesgue measure zero and meager subsets of $\mathbb{R}$, respectively. For the ideal $\mathcal{I} \in\{\mathcal{M}, \mathcal{N}\}$ its cofinality is defined by $\operatorname{cof}(\mathcal{I})=\min \{|\mathcal{B}|: \mathcal{B} \subset \mathcal{I}$ generates $\mathcal{I}\}$. A set $L \subset \mathbb{R}$ is a $\kappa$-Luzin set if $|L|=\kappa$ but $|L \cap N|<\kappa$ for every nowhere dense subset $N$ of $\mathbb{R}$. Recall that Martin's Axiom, MA, implies the existence of a $\mathfrak{c}$-Luzin set. The dominating number is defined as

$$
\mathfrak{d}=\min \left\{|T|: T \subset \omega^{\omega} \&\left(\forall f \in \omega^{\omega}\right)(\exists g \in T)(\forall n<\omega) f(n)<g(n)\right\} .
$$

It is well known that $\omega_{1} \leq \mathfrak{d} \leq \operatorname{cof}(\mathcal{N})$. (See e.g. [1].) In this paper we use term Polish space for a complete separable metric space without isolated points.

\section{On a Convergence of Subsequences}

This section can be viewed as an extension of the discussion around Egorov's theorem presented in [12, Ch. 9]. In 1932 Mazurkiewicz [13] proved the following variant of Egorov's theorem, where a sequence $\left\langle f_{n}\right\rangle_{n<\omega}$ of real-valued functions is uniformly bounded provided there exists an $r \in \mathbb{R}$ such that range $\left(f_{n}\right) \subset[-r, r]$ for every $n$.

Mazurkiewicz's Theorem Every uniformly bounded sequence $\left\langle f_{n}\right\rangle_{n<\omega}$ of real-valued continuous functions defined on a Polish space $X$ has a subsequence which is uniformly convergent on some perfect set $P$.

Of course Mazurkiewicz' theorem cannot be proved if we do not assume some regularity of the functions $f_{n}$ even if $X=\mathbb{R}$. But is it at least true that

(*) for every uniformly bounded sequence $\left\langle f_{n}: \mathbb{R} \rightarrow \mathbb{R}\right\rangle_{n<\omega}$ the conclusion of Mazurkiewicz' theorem holds for some $P \subset \mathbb{R}$ of cardinality $\mathfrak{c}$ ?

The consistency of the negative answer follows from the next example, which is essentially due to Sierpiński [16]. ${ }^{1}$ (See [12, pp. 193-194], where it is proved under the assumption of the existence of $\omega_{1}$-Luzin set. The same proof works also for our more general statement.)

Example 1. Assume that there exists a $\kappa$-Luzin set. Then for every $X$ of cardinality $\kappa$ there exists a sequence $\left\langle f_{n}: X \rightarrow\{0,1\}\right\rangle_{n<\omega}$ with the property that for every $W \in[\omega]^{\omega}$ the subsequence $\left\langle f_{n}\right\rangle_{n \in W}$ converges pointwise for less than $\kappa$-many points $x \in X$.

In particular, under Martin's Axiom the above sequence exists for every Polish space $X$ and $\kappa=\mathfrak{c}$.

\footnotetext{
${ }^{1}$ Sierpiński constructed this example under the assumption of the Continuum Hypothesis.
} 
Note also that under MA the above example can hold only for $\kappa=\mathfrak{c}$, since MA implies that

for every set $S$ of cardinality less than c every uniformly bounded sequence $\left\langle f_{n}: S \rightarrow \mathbb{R}\right\rangle_{n<\omega}$ has a pointwise convergent subsequence.

(See [12, p. 195].) Sharper results concerning the above two facts were recently obtained by Fuchino and Plewik [11], in which they relate them to the splitting number $\mathfrak{s}$. (For the definition of $\mathfrak{s}$ see e.g. [1]. For us it is only important that $\omega_{1} \leq \mathfrak{s} \leq \mathfrak{d}$.) More precisely, the authors show there that: For any $X \in[\mathbb{R}]^{<\mathfrak{s}}$ any sequence $\left\langle f_{n}: X \rightarrow[-\infty, \infty]\right\rangle_{n<\omega}$ has a subsequence convergent pointwise on $X$; however for any $X \in[\mathbb{R}]^{\mathfrak{s}}$ there exists a sequence $\left\langle f_{n}: X \rightarrow[0,1]\right\rangle_{n<\omega}$ with no pointwise convergent subsequence.

Our main goal of this section is to prove that $(*)$ is consistent with (so, by the example, also independent from) the usual axioms of set theory ZFC. To state this precisely we need the following terminology and facts.

A maximal non-principal filter $\mathcal{F}$ on $\omega$ is said to be Ramsey provided for every $B \in \mathcal{F}$ and $h:[B]^{2} \rightarrow\{0,1\}$ there exist $i<2$ and $A \in \mathcal{F}$ such that $A \subset B$ and $h\left[[A]^{2}\right]=\{i\}$. We say that a family $\mathcal{W} \subset \mathcal{F}$ generates filter $\mathcal{F}$ provided for every $F \in \mathcal{F}$ there exists a $W \in \mathcal{W}$ such that $W \subset F$.

Theorem 2. Assume that $\mathfrak{d}=\omega_{1}$ and there exists a Ramsey ultrafilter $\mathcal{F}$ on $\omega$ generated by a family $\mathcal{W} \subset \mathcal{F}$ of cardinality $\omega_{1}$.

Let $X$ be an arbitrary set and $\left\langle f_{n}: X \rightarrow \mathbb{R}\right\rangle_{n<\omega}$ be a sequence of functions such that the set $\left\{f_{n}(x): n<\omega\right\}$ is bounded for every $x \in X$. Then there are sequences: $\left\langle P_{\xi}: \xi<\omega_{1}\right\rangle$ of subsets of $X$ and $\left\langle W_{\xi} \in \mathcal{F}: \xi<\omega_{1}\right\rangle$ such that $X=\bigcup_{\xi<\omega_{1}} P_{\xi}$ and for every $\xi<\omega_{1}$ :

the sequence $\left\langle f_{n}\left\lceil P_{\xi}\right\rangle_{n \in W_{\xi}}\right.$ is monotone and uniformly convergent.

The conclusion of Theorem 2 is obvious for sets $X$ with cardinality $\leq \omega_{1}$, since sets $P_{\xi}$ can be chosen just as singletons. Thus, we will be interested in the theorem only for the sets $X$ of cardinality greater than $\omega_{1}$. If $X$ is a Polish space this leads to $\mathfrak{c}=|X|>\omega_{1}$. Luckily, the assumptions of Theorem 2 are consistent with ZFC+ "c $c=\omega_{2}$ ". This holds in the iterated perfect set model. More precisely, the fact that in this model we have $\mathfrak{c}=\omega_{2}$ and $\operatorname{cof}(\mathcal{N})=\omega_{1}$ can be found in $[1$, p. 339]. The fact that in this model there exists a desired Ramsey ultrafilter has been proved in Baumgartner, Laver [2]. (They proved there that there exists a selective $\omega_{1}$-generated ultrafilter on $\omega$. But it is well known that an ultrafilter on $\omega$ is selective if and only if it is Ramsey.) All these facts follow also from the axiom CPA, which is a subject of a forthcoming monograph [9]. (Some of the results proved here may also be included in [9] as the examples of interesting consequences of CPA.) 
In particular, we get the following corollary which, under additional set theoretical assumptions, generalizes Mazurkiewicz' theorem and implies $(*)$.

Corollary 3. It is consistent with $Z F C+" \mathfrak{c}=\omega_{2}$ " that for each Polish space $X$ and each uniformly bounded sequence $\left\langle f_{n}: X \rightarrow \mathbb{R}\right\rangle_{n<\omega}$ there exist sequences: $\left\langle P_{\xi}: \xi<\omega_{1}\right\rangle$ of subsets of $X$ and $\left\langle W_{\xi} \in[\omega]^{\omega}: \xi<\omega_{1}\right\rangle$ such that $X=\bigcup_{\xi<\omega_{1}} P_{\xi}$ and for every $\xi<\omega_{1}$ :

the sequence $\left\langle f_{n}\left\lceil P_{\xi}\right\rangle_{n \in W_{\xi}}\right.$ is monotone and uniformly convergent.

In particular, there exists a $\xi<\omega_{1}$ such that $\left|P_{\xi}\right|=\mathfrak{c}$.

Moreover, if functions $f_{n}$ are continuous then we can additionally require that all sets $P_{\xi}$ are closed in $X$.

Proof. The main part follows immediately from the discussion above and the Pigeon Hole Principle. To see the additional part it is enough to note that for continuous functions sets $P_{\xi}$ can be replaced by their closures, since for any sequence $\left\langle f_{n}: P \rightarrow \mathbb{R}\right\rangle_{n<\omega}$ of continuous functions if $\left\langle f_{n}\lceil D\rangle_{n<\omega}\right.$ is monotone and uniformly convergent for some dense subset $D$ of $P$ then so is $\left\langle f_{n}\right\rangle_{n<\omega}$.

Proof of Theorem 2. For every $x \in X$ define $h_{x}:[\omega]^{2} \rightarrow\{0,1\}$ by putting for every $n<m<\omega$

$$
h_{x}(n, m)=1 \text { if and only if } f_{n}(x) \leq f_{m}(x) .
$$

Since $\mathcal{F}$ is Ramsey and $\mathcal{W}$ generates $\mathcal{F}$ we can find a $W_{x} \in \mathcal{W}$ and an $i_{x}<2$ such that $h_{x}\left[\left[W_{x}\right]^{2}\right]=\left\{i_{x}\right\}$. Thus, the sequence $S_{x}=\left\langle f_{n}(x)\right\rangle_{n \in W_{x}}$ is monotone. It is increasing when $i_{x}=1$ and it is decreasing for $i_{x}=0$.

For $W \in \mathcal{W}$ and $i<2$ let $P_{W}^{i}=\left\{x \in X: W_{x}=W \& i_{x}=i\right\}$. Then $\left\{P_{W}^{i}: W \in \mathcal{W} \& i<2\right\}$ is a partition of $X$ and for every $W \in \mathcal{W}$ and $i<2$ the sequence $\left\langle f_{n}\left\lceil P_{W}^{i}\right\rangle_{n \in W}\right.$ is monotone and pointwise convergent to some function $f: P_{W}^{i} \rightarrow \mathbb{R}$.

To get uniform convergence note that for every $x \in P_{W}^{i}$ there exists an $s_{x} \in \omega^{\omega}$ such that

$$
(\forall k<\omega)\left(\forall n \in W \backslash s_{x}(k)\right)\left|f_{n}(x)-f(x)\right|<2^{-k} .
$$

Since $\mathfrak{d}=\omega_{1}$, there exists a $T \in\left[\omega^{\omega}\right]^{\omega_{1}}$ dominating $\omega^{\omega}$. In particular, for every $x \in P_{W}^{i}$ there exists a $t_{x} \in T$ such that $s_{x}(n) \leq t_{x}(n)$ for all $n<\omega$. For $t \in T$ let

$$
P_{W}^{i}(t)=\left\{x \in P_{W}^{i}: t_{x}=t\right\} .
$$

Since every sequence $\left\langle f_{k}\left\lceil P_{W}^{i}(t)\right\rangle_{k \in W}\right.$ is monotone and uniformly convergent, $\left\{P_{W}^{i}(t): i<2, W \in \mathcal{W}, t \in T\right\}$ is the desired covering $\left\{P_{\xi}: \xi<\omega_{1}\right\}$ of $X$. 


\section{$2 \operatorname{cof}(\mathcal{N})=\omega_{1}$, Blumberg Theorem, and Magic Set}

In this section we will show two consequences of $\operatorname{cof}(\mathcal{N})=\omega_{1}$.

In 1922 Blumberg [4] proved that for every $f: \mathbb{R} \rightarrow \mathbb{R}$ there exists a dense subset $D$ of $\mathbb{R}$ such that $f \uparrow D$ is continuous. This theorem sparked a lot of discussion and generalizations, see e.g. [7, pp. 147-150]. In particular, Shelah [15] showed that there is a model of ZFC in which for every $f: \mathbb{R} \rightarrow \mathbb{R}$ there is a nowhere meager subset $D$ of $\mathbb{R}$ such that $f \uparrow D$ is continuous. The dual measure result, that is the consistency of a statement for every $f: \mathbb{R} \rightarrow \mathbb{R}$ there is a subset $D$ of $\mathbb{R}$ of positive outer Lebesgue measure such that $f\lceil D$ is continuous, has been also recently established by Rosłanowski and Shelah [14]. Below we note that each of these properties contradicts $\operatorname{cof}(\mathcal{N})=\omega_{1}$. (We use here the well known inequality $\operatorname{cof}(\mathcal{M}) \leq \operatorname{cof}(\mathcal{N})$. See e.g. [1].)

Theorem 4. Let $\mathcal{I} \in\{\mathcal{N}, \mathcal{M}\}$. If $\operatorname{cof}(\mathcal{I})=\omega_{1}$ then there exists an $f: \mathbb{R} \rightarrow \mathbb{R}$ such that $f \uparrow D$ is discontinuous for every $D \in \mathcal{P}(\mathbb{R}) \backslash \mathcal{I}$.

Proof. We will assume that $\mathcal{I}=\mathcal{N}$, the proof for $\mathcal{I}=\mathcal{M}$ being essentially identical.

Let $\left\{N_{\xi} \subset \mathbb{R}^{2}: \xi<\omega_{1}\right\}$ be a family cofinal in the ideal of null subsets of $\mathbb{R}^{2}$ and for each $\xi<\omega_{1}$ let

$$
N_{\xi}^{*}=\left\{x \in \mathbb{R}:\left(N_{\xi}\right)_{x} \notin \mathcal{N}\right\},
$$

where $\left(N_{\xi}\right)_{x}=\left\{y \in \mathbb{R}:\langle x, y\rangle \in N_{\xi}\right\}$. By Fubini's theorem each $N_{\xi}^{*}$ is null. For each $x \in N_{\xi}^{*} \backslash \bigcup_{\zeta<\xi} N_{\zeta}^{*}$ we choose $f(x)$ so that

$$
f(x) \notin \bigcup_{\zeta<\xi}\left(N_{\zeta}\right)_{x} .
$$

Then function $f$ is as desired.

Indeed, if $f \nmid D$ is continuous for some $D \subset \mathbb{R}$ then $f \uparrow D$ is null in $\mathbb{R}^{2}$. In particular, there exists a $\xi<\omega_{1}$ such that $f \uparrow D \subset N_{\xi}$. But this means that $D \subset \bigcup_{\zeta \leq \xi} N_{\zeta}^{*}$.

Note that essentially the same proof works if we assume only that $\operatorname{cof}(\mathcal{I})$ is equal to the additivity number $\operatorname{add}(\mathcal{I})$ of $\mathcal{I}$.

Corollary 5. Assume $\operatorname{cof}(\mathcal{N})=\omega_{1}$. Then there exists an $f: \mathbb{R} \rightarrow \mathbb{R}$ such that if $f \uparrow D$ is continuous then $D \in \mathcal{N} \cap \mathcal{M}$.

Proof. Let $f_{\mathcal{N}}$ and $f_{\mathcal{M}}$ be from Theorem 4 constructed for the ideals $\mathcal{N}$ and $\mathcal{M}$, respectively. Let $G \subset \mathbb{R}$ be a dense $G_{\delta}$ of measure zero and put $f=\left[f_{\mathcal{M}}\lceil G] \cup\left[f_{\mathcal{N}}\lceil(\mathbb{R} \backslash G)]\right.\right.$. Then this $f$ is as desired. 
Recall that a set $M \subset \mathbb{R}$ is a magic set (or set of range uniqueness) if for every different nowhere constant functions $f, g \in C(\mathbb{R})$ we have $f[M] \neq g[M]$. It has been proved by Berarducci and Dikranjan [3, thm. 8.5] that a magic set exists under $\mathrm{CH}$. We like to note here that the same is implied by a much weaker assumption that $\operatorname{cof}(\mathcal{M})=\omega_{1}$. However, the existence of a magic set is independent of ZFC, as proved by Ciesielski and Shelah in [10].

Proposition 6. If $\operatorname{cof}(\mathcal{M})=\omega_{1}$ then there exists a magic set.

Proof. An uncountable set $L \subset \mathbb{R}$ is a 2-Luzin set provided for every disjoint subsets $\left\{x_{\xi}: \xi<\omega_{1}\right\}$ and $\left\{y_{\xi}: \xi<\omega_{1}\right\}$ of $L$, where the enumerations are oneto-one, the set of pairs $\left\{\left\langle x_{\xi}, y_{\xi}\right\rangle: \xi<\omega_{1}\right\}$ is not a meager subset of $\mathbb{R}^{2}$. In [5, prop. 4.8] it was noticed that every $\omega_{1}$-dense 2 -Luzin set is a magic set. It is also a standard and easy diagonal argument that $\operatorname{cof}(\mathcal{M})=\omega_{1}$ implies the existence of a $\omega_{1}$-dense 2-Luzin set. (The proof presented in [17, prop. 6.0] works also under the assumption $\operatorname{cof}(\mathcal{M})=\omega_{1}$.) So, $\operatorname{cof}(\mathcal{M})=\omega_{1}$ implies that there is a magic set.

Recall also that the existence of a magic set for the class $D^{1}$ of all differentiable functions can be proved in ZFC. This follows from [6, thm. 3.1], since every function from $D^{1}$ belongs to the class $\left(T_{2}\right)$. (Compare also [6, cor. 3.3 and 3.4].)

\section{References}

[1] T. Bartoszyński, H. Judah, Set Theory, A K Peters, 1995.

[2] J. Baumgartner, R. Laver, Iterated perfect-set forcing, Ann. Math. Logic 17 (1979), 271-288.

[3] A. Berarducci, D. Dikranjan, Uniformly approachable functions and UA spaces, Rend. Ist. Matematico Univ. di Trieste 25 (1993), 23-56.

[4] H. Blumberg, New properties of all real functions, Trans. Amer. Math. Soc. 24 (1922), 113-128.

[5] M. R. Burke, K. Ciesielski, Sets on which measurable functions are determined by their range, Canad. J. Math. 49 (1997), 1089-1116. (Preprint* available. $^{2}$ )

[6] M. R. Burke, K. Ciesielski, Sets of range uniqueness for classes of continuous functions, Proc. Amer. Math. Soc. 127 (1999), 3295-3304. (Preprint* available.)

\footnotetext{
${ }^{2}$ Preprints marked by * are available in electronic form from Set Theoretic Analysis Web Page: http://www.math.wvu.edu/ ${ }^{\sim}$ kcies/STA/STA.html
} 
[7] K. Ciesielski, Set theoretic real analysis, J. Appl. Anal. 3(2) (1997), 143190. (Preprint ${ }^{\star}$ available.)

[8] K. Ciesielski, Set Theory for the Working Mathematician, London Math. Soc. Stud. Texts 39, Cambridge Univ. Press 1997.

[9] K. Ciesielski, J. Pawlikowski, Covering property axiom CPA, work in progress ${ }^{\star}$.

[10] K. Ciesielski, S. Shelah, Model with no magic set, J. Symbolic Logic 64(4) (1999), 1467-1490. (Preprint* available.)

[11] S. Fuchino, Sz. Plewik, On a theorem of E. Helly, Proc. Amer. Math. Soc. 127(2) (1999), 491-497.

[12] A. B. Kharazishvili, Strange Functions in Real Analysis, Pure and Applied Mathematics 229, Marcel Dekker, 2000.

[13] S. Mazurkiewicz, Sur les suites de fonctions continues, Fund. Math. 18 (1932), 114-117.

[14] A. Rosłanowski, S. Shelah, Measured Creatures, preprint.

[15] S. Shelah, Possibly every real function is continuous on a non-meagre set, Publ. Inst. Mat. (Beograd) (N.S.) 57(71) (1995), 47-60.

[16] W. Sierpiński, Remarque sur les suites infinies de fonctions (Solution d'un problème de M. S. Saks), Fund. Math. 18 (1932), 110-113.

[17] S. Todorcevic, Partition problems in topology, Contemp. Math. 84, Amer. Math. Soc. 1989. 